\title{
Exploring the superadditive effects of scent and pictures on verbal recall: An extension of dual coding theory ${ }^{\text {tr }}$
}

\author{
May O. Lwin ${ }^{a, 1}$, Maureen Morrin ${ }^{\text {b,*,1 }}$, Aradhna Krishna ${ }^{\text {c, } 1}$ \\ a Nanyang Technological University, Singapore \\ ${ }^{\mathrm{b}}$ Rutgers University, Camden, NJ, USA \\ ${ }^{\mathrm{c}}$ University of Michigan, Ann Arbor, MI, USA
}

Received 25 February 2009; revised 17 March 2010; accepted 4 April 2010

Available online 7 May 2010

\begin{abstract}
This research extends the dual coding theory of memory retrieval (Paivio 1969, 2007) beyond its traditional focus on verbal and pictorial information to olfactory information. We manipulate the presence or absence of olfactory and pictorial stimuli at the time of encoding (study 1 ) or retrieval (study 2) and measure the impact on verbal recall. After a time delay, scent enhances recall of verbal information, and scent-based retrieval cues potentiate the facilitative effect of pictures on recall. These results cannot be attributed merely to increased elaboration at the time of exposure.
\end{abstract}

(C) 2010 Society for Consumer Psychology. Published by Elsevier Inc. All rights reserved.

Keywords: Dual coding theory; Sensory; Scent; Pictures; Memory

\section{Introduction}

Consumers are exposed to a plethora of verbal information about products in print and Internet advertisements (ads), on billboards and packages, and on products themselves. At the same time, marketers' use of multisensory stimuli in communications about products appears to be on the rise. For example, print ads can have specially textured paper to replicate a sandy beach, embed product scents in the print ad itself, and even provide taste-test strips (e.g., firstflavor.com). In the expanding realm of possibilities created by our fast-evolving technological environment, such multisensory experiences add a new dimension to product and communication offerings and present the possibility of significantly enhancing the consumer's experience.

\footnotetext{
is The authors would like to thank the following students for their help in this project: Shelly Malik, Mindawati Wijaya, Grace Chiang Pei Sze, Kenneth Goh, Beverly Maria Snodgrass, and Adrian Yeap Shiqiang, of Nanyang Technological University.

* Corresponding author.

E-mail address: mmorrin@rutgers.edu (M. Morrin).

${ }^{1}$ The authors contributed equally to this work.
}

The bulk of consumer research, however, has focused on how consumers respond just to verbal information. More recently, the focus seems to be expanding to a consideration of the combined impact of both verbal and visual information (Bagozzi, 2008; Childers \& Jiang, 2008; Heckler \& Childers, 1992; Houston, Childers \& Heckler, 1987; MacInnis \& Price, 1987; Wyer, Hung \& Jiang, 2008a, Wyer, Hung \& Jiang, 2008b). Still, relatively little attention is paid to the information processing implications of acoustic, haptic, gustatory, and olfactory sensory input, especially when combined with input from the other sensory modalities. Bagozzi (2008, p. 263) specifically calls for such efforts: "How odors, in particular, and sensory processes, in general, function in comprehension and decision making, when information is presented in visual, verbal, olfactory, and other sensory modes, are fruitful areas for future research." Heeding this call, we contribute to the literature on consumer information processing by identifying when multisensory input that includes olfactory input will enhance recall. We move beyond a focus on verbal and visual information (Wyer et al., 2008a,b) and introduce a third cue, scent. In doing so, we raise the possibility that three separate systems of memory may enhance recall, and we identify when that occurs. We 
propose that olfactory and pictorial stimuli have different types of effects on memory over time and that the effects can be not only additive but also superadditive in nature.

The rest of the paper is organized as follows: we discuss our conceptual framework within the context of prior research, and the results of two studies. Then we provide conclusions and ideas for future research.

\section{Conceptual framework}

\section{Dual coding theory (DCT) and synergistic memory effects}

A critical issue we explore in this research is the potential for synergistic effects between pictorial and olfactory input on memory for verbal information. For example, if pictures increase verbal memory by $x$ units, and scent increases it by $y$ units, and the presence of both pictures and scent increase memory by more than $x+y$ units, then the two sensory inputs would be working in more than an additive fashion-they would be working in a superadditive fashion. When might we observe such an effect? We develop an argument for superadditive effects based on scent's ability to enhance the imagery value of the memory trace - an extension of dual coding theory.

The psychological work on multisensory inputs has focused largely on concurrent visual and written verbal information (Paivio, 1969, 2007). The primary framework for such work, dual coding theory (DCT; Paivio, 1969, 1971, 1986, 2007), suggests that pictures enhance memory for verbal (written) information because humans process written information and pictures via two independent cognitive subsystems: one devoted to verbal information and one to imagery-based information. This is consistent with MacInnis and Price's (1987) distinction between discursive (i.e., symbolic, language-like) and imagery-based information processing. Per DCT, the verbal system processes incoming information sequentially, whereas the imaginal system processes it more holistically (Paivio, 1986, 2007). Researchers have also conceptualized the difference between the two forms of information in terms of hemispheric lateralization, noting that the processing of verbal information exhibits left-brain dominance (e.g., McKeever \& Hulling, 1971), whereas the processing of imaginal information exhibits right-brain dominance (e.g., Rizzolatti, Umilta \& Berlucchi, 1971). More recently, brain imaging studies confirm that verbal and visual information tends to be processed in different areas of the brain (Childers \& Jiang, 2008). The verbal and imaginal systems are proposed to function largely independently, but they have the capacity to interact to enhance memory performance.

\section{The additivity hypothesis}

The additivity hypothesis of DCT, which has received considerable empirical support, proposes that verbal plus imaginal information input will have additive effects on memory retrieval (Begg, 1972). This means that more items of the verbal information will be remembered if pictorial stimuli accompany the presentation of the verbal information. The basic notion is that the effects are additive because the verbal and imaginal systems are largely independent. When incoming information is encoded via both verbal and imaginal codes, the verbal and imaginal memory traces are linked, so that, during attempts at retrieval, activation levels are pooled, enhancing the accessibility of the information. Numerous studies have confirmed that pictorial information improves memory for verbal information (e.g., Anderson \& Bower, 1973; cf. Wyer et al., 2008b). In this research, we extend the scope of DCT beyond its traditional focus on verbal and visual information to encompass an additional sensory input, specifically olfactory information.

In DCT, imagery is conceived of as a special form of representation in memory, in that it speeds the knowledge acquisition process by "concretizing" or making more concrete verbal or language-based information (Paivio, 2007). This concretization principle from DCT is used to explain why concrete phrases such as "white horse" are recalled at twice the rate of abstract phrases such as "basic truth" (Begg, 1972). To the extent that a retrieval cue is more concrete than abstract, recall is also enhanced (Paivio, 2007). Concrete cues are those more capable of generating a visual image and, therefore, more effective at facilitating recall because they are more capable of bringing to mind the object or event stored in a memory trace.

It stands to reason that sensory input that helps one concretize an object or event should help to activate the related symbolic or verbal information from memory (Paivio, 2007). As MacInnis and Price (1987, p. 474) note, "The evocation of imagery may be multisensory-involving images that incorporate, for example, smell, taste, sight, and tactile sensations - or may involve a single sensory dimension such as sight." Thus, imagery does not necessarily refer simply to visual imagery but rather to a concrete evocation that can consist of multisensorial dimensions. Since imagery is defined as a process by which all sensory information is represented in working memory (MacInnis \& Price, 1987, p. 473), olfactory sensory input may indeed have the capacity to increase a memory trace's imagery value. Thus, scent may not only enhance memory but also facilitate the ability of visual images to enhance memory.

Paivio (2007; see also Thompson \& Paivio, 1994) proposes that the different sensory modalities should have additive effects on recall if they are functionally independent from the symbolic (i.e., verbal) modality. However, Paivio alludes to the possibility of superadditive effects (Paivio, 2007, p. 167). He states additional research is needed to see whether the various sensory modalities of an object contribute additively or superadditively to recall (Paivio, 2007, p. 171).

\section{Superadditivity}

What effect will adding a third stimulus input, from a different sensory modality (i.e., olfaction), have on memory for verbal information? We propose that scent will have additive effects to the extent it is stored independently in its own code and superadditive effects to the extent that the olfactory information interacts with or activates information from the other senses (e.g., visual imagery).

Some researchers have suggested that the sense of smell possesses its own, relatively independent, nonverbal, olfactory code (Bensafi et al., 2003; Engen, 1987; Lyman \& McDaniel, 1990). If an additional sensory input (i.e., scent) has a largely independent memory code, orthogonal to the verbal and pictorial memory codes, then it is likely to exhibit additive effects on 
memory. If such a result is obtained, it would suggest that olfactory sensory input will enhance memory for verbal information in a manner similar to that of pictorial information, essentially extending the additivity hypothesis of dual coding theory to the realm of olfactory input. In other words, dual coding theory would be applicable not only to verbal + pictorial stimuli but also to verbal+ pictorial+olfactory stimuli. An alternative is that olfactory input, besides having an independent olfactory memory trace, interacts with other sensory aspects of the imagery value of the memory trace (e.g., visual, tactile, etc.). If so, olfactory input could have the capability of potentiating (i.e., increasing the facilitative effect on memory of) the other sensory inputs and exhibit superadditive effects on memory.

We propose that whether scent is additive or superadditive in the presence of pictures will be a function of the extent to which scent enhances not only the olfactory dimension of a memory trace's imagery level but also the visual dimension. If a scent-based retrieval cue merely increases the extent to which a consumer can imagine what a product smells like (i.e., olfactory imagery), we would expect to observe additive effects on memory performance (i.e., an additional cue from a different sensory modality should increase how much is remembered). If a scent-based retrieval cue also increases the extent to which a consumer can imagine what a product looks like (i.e., visual imagery), we would expect to observe superadditive effects on memory in the presence of pictures (i.e., scent would then have the capability of also increasing the effectiveness of picture cues on retrieval).

We next discuss when we expect to observe such effects.

\section{Scent and memory}

Recent and continuing research in the basic sciences suggests that there are aspects of the memory consolidation process that are uniquely associated with the sense of smell (Gerber \& Menzel, 2000). Neuroscientific research suggests that the memory consolidation process associated with olfaction occurs in multiple stages, with an immediate preliminary stage performed in parts of the olfactory bulb and brain just after stimulus exposure, followed by a later consolidation effort implicating other parts of the brain and resulting in deeper and longer-lasting memory traces (Gerber \& Menzel, 2000; Sanchez-Andrade, James \& Kendrick, 2005).

Consistent with these neuroscientific findings are patterns of memory for scent over time. Research shows, for example, that scent information exhibits lower initial acquisition levels than information obtained via the other senses (Zucco, 2003). So, for example, when tested immediately after exposure, a person might recognize 7 out of 10 scents smelled, compared to 9 out of 10 pictures seen or words read (Zucco, 2003, p. 156). However, once information has been encoded via the sense of smell into long-term memory, it is particularly long-lasting. Odor recognition studies show that people's ability to recognize scents they have encountered previously persists over very long periods, with minimal reductions in recognition accuracy from seconds (Engen, Kuisma, \& Eimas, 1973) to months or years after exposure (Engen, \& Ross, 1973; Zucco, 2003). Memories for other sensory inputs decay at a much faster rate, exhibiting steep forgetting curves
(Ebbinghaus, 1885). For instance, recognition accuracy for pictures dropped from $99 \%$ when measured immediately after exposure to $58 \%$ when measured 4 months later (Shepard, 1967). Memories for scents thus exhibit flatter forgetting curves over time than do memories for information acquired via the other sensory modalities.

These observations suggest that, compared to the other sensory modalities, the sense of smell may be particularly effective at enhancing memory for associated information in the long-term. If, indeed, scent's impact on memory performance is more evident after a time delay, it seems likely that its ability to potentiate pictures' effects on memory also emerges after a time delay.

We state our expectations more formally as:

H1. Scent-based retrieval cues will potentiate the effect of pictorial stimuli on memory for verbal information (i.e., exhibit superadditive effects) after a time delay.

H2. Scent-based retrieval cues will enhance the visual imagery value of an object's memory trace.

\section{Overview of studies}

In our studies, we replicate real-world scenarios as closely as possible while also attempting to control for the influence of extraneous variables. Both studies focus on verbal, pictorial, and olfactory information presented in print advertisements via direct mailers. In study 1 , we manipulate the presence or absence of pictures and scent at the time of exposure to the product and advertisement (i.e., at encoding); in study 2, we manipulate the presence or absence of pictures and scent when memory is tested (i.e., at retrieval), which allows us to rule out the possibility that the observed effects are attributable merely to differences in the amount of cognitive elaboration at encoding. To summarize, we expect to find that scent will potentiate the effect of pictures on recall after a time delay $(\mathrm{H} 1)$ and that scent will enhance not only olfactory but also visual imagery (H2). Moreover, we expect to find these effects even when we control for stimulus elaboration.

We now present the empirical results.

\section{Study 1: sensory input manipulated at encoding}

\section{Pretests}

We conducted two pretests to select a product category, a product scent, and a hypothetical brand name for the stimuli to be used in the two studies. On the basis of a small-scale initial pretest $(n=13)$, we identified a rose/sandalwood-scented moisturizer product with a hypothetical name of either Levadia or Florica as the stimuli that would best serve our purpose. We then conducted a second, larger pretest among 32 respondents (mean age $=28$ years, $69 \%$ female) to make a final determination of the hypothetical brand name, product category, and scent to be used in the main studies. Participants rated seven brand names (Evian, Levadia, Novara, San Remo, Florica, Ivrea, and Sony) on familiarity (from $1=$ not at all familiar to $5=$ very familiar) and appropriateness for a 
moisturizer product (from $1=$ not at all appropriate to $5=$ very appropriate).

Five scent combinations were also tested (rose/sandalwood, ylang-ylang/lemon, lavender/ginger flower, eucalyptus/rosemary, and lavender/ginger flower). A few drops of each scent blend were placed onto each end of long strips of white litmus paper, which were then placed into cups identified by randomly assigned letters (A, B, C, D, and E; method modeled after Spangenberg et al., 1996). The cups were presented to participants in random order. They were instructed to pick up the litmus paper to sniff the sample about $10 \mathrm{~cm}$ from the nose, as many times as they liked. They were asked to rate each of the scents on the basis of pleasantness (from $1=$ very unpleasant to $5=$ very pleasant), strength (from $1=$ weakly scented to $5=$ strongly scented), and appropriateness for a moisturizer product (from $1=$ very inappropriate to $5=$ very appropriate). Participants were permitted to smell the scent samples as often as they liked and in any order they wished while answering these questions. Finally, they were asked how important scent would be in influencing their evaluation of various types of products (from $1=$ not at all important to $5=$ very important). Participants also filled out gender and age questions.

Scent was rated as significantly more important in influencing evaluation for a moisturizer $(M=4.63)$ and spa package $(M=4.72)$ than for any of the other products tested (pen $M=1.59$, wallet $M=2.19$, comb $M=2.00$, laptop $M=1.53$, camera $M=1.34$; all paired $t$ tests vs. moisturizer or spa package $p$ values $<.01)$. There was no difference in importance between the spa package and moisturizer (paired $t=1.00, p>.30$ ). We decided to use moisturizer as the product.

Participants were most familiar with the actual brands (e.g., Sony $M=4.56$ and Evian $M=4.13$ ) and least familiar with the hypothetical brands Levadia $(M=1.16)$ and Ivrea $(M=1.16)$. Levadia and Ivrea were considered about equally appropriate names for a moisturizer product ( 2.65 vs. 3.00 , paired $t=1.50$, $p>.10)$. We chose Levadia as the hypothetical brand name for use in the studies, based on the results of both pretests.

All the five scent combinations were rated as being fairly pleasant (range: $M=1.75$ to $M=3.34$ ), but the rose/sandalwood scent combination was rated as most pleasant $(M=3.34)$, as in the first pretest. The rose/sandalwood scent was also rated as most appropriate for a moisturizer product $(M=3.47)$ compared to the other scents ( $M=1.47$ to $M=3.31$; significantly different only from lavender/ginger flower, $p<.01)$. The rose/sandalwood scent was also perceived to be moderately strong $(M=3.66)$, falling about in the middle of the range of perceived scent strengths (which ranged from $M=2.22$ to $M=4.41$ ). Thus, we chose rose/sandalwood as the scent for the main studies.

\section{Sample and design}

One hundred females were recruited for participation in study 1. Prior research suggests that gender differences in scent preferences may exist (Spangenberg, Sprott, Grohmann \& Tracy, 2006). As such, we limited ourselves to only females in this study so as to have greater experimental control (note: we relax this restriction in study 2). Participants were provided with a token gift of packed candy at the completion of the experiment.
The study had a scent (direct mailer scented: yes, no) $\times$ image (direct mailer contained floral image: yes, no) $\times$ time delay (memory tested after: short delay, long delay) mixed design. The scent and image factors were manipulated on a between-subjects basis. The floral image had a vivid yellow theme, and the scent used was rose/sandalwood. Each participant was randomly assigned to one of the four cells of the experimental design. Time delay was manipulated on a within-subjects basis, with all participants' memories tested after both a short ( 5 minutes after exposure) and long ( 2 weeks after exposure) time delay.

\section{Stimulus materials}

Two versions of a full-color, $8.5 \times 11$-inch direct mailer insert describing a hypothetical brand of moisturizer were created. Both versions of the insert contained the brand name plus several copy points on the right side of the sheet of paper. In the no image condition, the left side contained only a picture of the moisturizer bottle (see Fig. 1a). In the image condition, the left side contained a picture of the moisturizer bottle plus a floral visual at both the top and the bottom (see Fig. 1b). Also, in the image condition, the floral visual was repeated on the outside of the envelope in which the insert was enclosed, whereas in the no image condition, blank envelopes were used. The yellow floral visual, obtained from an online image library, was chosen because we believed it to be reasonably congruent with the product and thus thought it would not interfere with the participants' motivation to process the other information in the ad (Miniard et al., 1991).

To infuse the direct mailer insert with the rose/sandalwood scent, 2-3 drops of the scent blend were dripped onto the inserts and left overnight (for 12 hours) to permeate the papers thoroughly. The inserts were then placed into envelopes, either with or without the image according to image condition. We attempted to use an amount of scent blend that, upon opening the envelope, would be noticeable but not overwhelming.

For the aided retrieval parts of the experiment, white (no image) or yellow (image) litmus paper was used that either was or was not infused with the rose/sandalwood scent. Those in the no scent condition received an unscented piece of paper; those in the scented condition received a scented piece of paper. Similarly, those in the image condition received yellow litmus paper (same shade as the floral image) and those in the no image condition received white litmus paper, to yield four different types of retrieval aids to match the four between-subjects conditions experienced at the time of encoding.

\section{Procedure}

Participants took part in the first phase of the study one at a time. They were told that the purpose of the study was to investigate "the use of different stimuli in advertising" and were informed they would need to return after 2 weeks for another, unrelated experiment. They were unaware that their memories would be tested.

Participants were given an envelope containing a direct mailer insert and were asked to inspect it as they might if they 
(a)

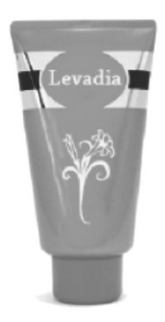

(b)
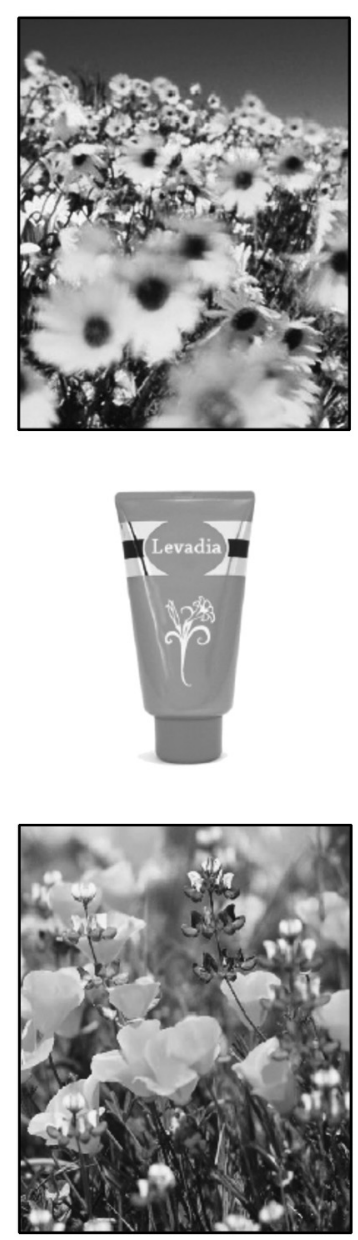

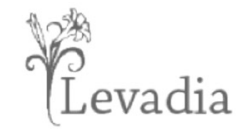

Nourish your skin with Levadia, the moisturiser that makes your skin baby soft.

Made from $100 \%$ pure plant extracts, Levadia blends soothing floral essential oils and exotic herbs with Vitamin $\mathbf{C}$ that leaves your skin smooth to the touch.

Levadia helps to whiten your skin by allowing new skin cells to emerge.

Awaken your senses with a refreshing experience today.

Only with Levadia.

At a promotional price of $\mathbf{\$ 9 . 9 0}$

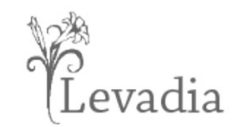

Nourish your skin with Levadia, the moisturiser that makes your skin baby soft.

Made from $100 \%$ pure plant extracts, Levadia blends soothing floral essential oils and exotic herbs with Vitamin $\mathbf{C}$ that leaves your skin smooth to the touch.

Levadia helps to whiten your skin by allowing new skin cells to emerge.

Awaken your senses with a refreshing experience today.

Only with Levadia.

At a promotional price of $\$ 9.90$ had received it in the mail. When finished inspecting the direct mailer, participants raised their hands and an experimenter took the direct mailer from them. After a 5-minute delay during which participants were given filler tasks involving looking at other unrelated brochures (one for a credit card and one for a university fund raiser), the experimenter gave them a survey booklet to complete. They answered questions in the booklet, including those assessing unaided recall. They were asked specifically, "What is the brand name of the moisturizer?" and "Please write down everything you can remember about the moisturizer." When done, they were provided with a retrieval cue (the litmus paper) and asked to remember anything else they could about the advertised product. Two weeks after the first phase of the experiment, the participants returned to again complete the unaided and aided recall parts of the survey, as in phase 1. Participants then completed some demographic questions. They were debriefed and thanked for their participation.

\section{Results}

Memory for verbal information was measured in two ways - unaided and incremental aided recall (see Table 1 and Fig. 2). To calculate unaided recall, we summed the number of correct verbal attributes remembered before provision of the sensory-based retrieval cue (of 13 possible plus the brand name $=14$ total possible score). Incremental aided recall was measured as the number of incremental items of information recalled after the sensory retrieval cue was provided (thus, if an item was recalled both without and with the retrieval cue, it was not counted again in incremental aided recall). The unaided and incremental aided recall scores were calculated separately for the two periods when memory was tested (i.e., 5 minutes after exposure and after a 2-week delay). All coding was conducted by two independent coders and had a 95\% agreement rate. Any disagreements between the coders were resolved via discussion.

\section{Unaided recall}

We conducted a repeated-measures MANOVA on unaided recall as a function of the between-subjects factors of scent (yes, no) and image (yes, no) and the within-subjects factor of time delay (short, long). Looking first at between-subject effects, we obtain significant main effects for scent $\left(F(1,96)=31.41, p<.01, \eta^{2}=.25\right.$; $M_{\text {NoScent }}=2.38$ vs. $\left.M_{\text {Scent }}=3.54\right)$ and image $(F(1,96)=15.70$, $p<.01, \eta^{2}=.14 ; M_{\text {NoImage }}=2.55$ vs. $\left.M_{\text {Image }}=3.37\right)$, with both increasing unaided recall by about 1 piece of information.

Within-subject effects reveal a main effect of delay $(F(1,96)=$ $244.53, p<.01, \eta^{2}=.72 ; M_{\text {Short-term }}=4.23$ vs. $\left.M_{\text {Long-term }}=1.69\right)$, with participants forgetting nearly 3 pieces of information over 2 weeks, as might be expected. We also obtain a scent by delay interaction $\left(F(1,96)=33.49, p<.01, \eta^{2}=.26\right)$, which, as described next, suggests that scent was more effective at enhancing memory after a long time delay. After a short delay (5 minutes), scent did not enhance unaided recall $(p>0.4)$, whereas image did so marginally $\left(F(1,96)=3.62, p<.1, \eta^{2}=.04 ; M_{\text {NoImage }}=3.94\right.$ vs. $\left.M_{\text {Image }}=4.52\right)$. After a long time delay, however, we find significant main effects for both scent $\left(F(1,96)=97.14, p<.01, \eta^{2}=.50 ; M_{\text {NoScent }}=0.64\right.$

Fig. 1. Direct mailer inserts. (a) No image. (b) Image. 
Table 1

Study 1 direct mailer: mean recall by condition ${ }^{\mathrm{a}}$.

\begin{tabular}{|c|c|c|c|c|c|c|c|c|}
\hline & \multicolumn{4}{|l|}{ Short delay } & \multicolumn{4}{|l|}{ Long delay } \\
\hline & \multicolumn{2}{|l|}{ No scent } & \multicolumn{2}{|l|}{ Scent } & \multicolumn{2}{|l|}{ No scent } & \multicolumn{2}{|l|}{ Scent } \\
\hline & Low image & High image & Low image & High image & Low image & High image & Low image & High image \\
\hline Unaided recall & $3.92(0.16)$ & $4.32(0.20)$ & $3.96(0.24)$ & $4.72(0.24)$ & $0.08(0.00)$ & $1.20(0.28)$ & $2.24(0.24)$ & $3.24(0.32)$ \\
\hline Incremental aided recall & $0.00(0.00)$ & $0.60(0.72)$ & $0.44(0.28)$ & $1.20(0.84)$ & $0.00(0.00)$ & $0.20(0.12)$ & $0.60(0.32)$ & $1.88(1.44)$ \\
\hline Total recall & $3.92(0.16)$ & $4.92(0.92)$ & $4.40(0.52)$ & $5.92(1.08)$ & $0.08(0.00)$ & $1.40(0.40)$ & $2.84(0.56)$ & $5.12(1.76)$ \\
\hline
\end{tabular}

${ }^{a}$ Numbers in cells are mean number of items recalled out of a total possible of 14: Levadia, moisturizer, nourishes, baby soft, $100 \%$ pure, plant extracts, floral, essential oils/herbs, vitamin C, smooth skin, whitening, refreshing, promotional price, $\$ 9.90$. Numbers in parentheses are mean number of floral imagery items recalled.

vs. $\left.M_{\text {Scent }}=2.74\right)$ and image $\left(F(1,96)=24.75, p<.01, \eta^{2}=.20\right.$; $M_{\text {NoImage }}=1.16$ vs. $\left.M_{\text {Image }}=2.22\right)$, with scent exhibiting an effect size more than double that of image $\left(\eta^{2}=.50\right.$ vs. $\left.\eta^{2}=.20\right)$. The pattern of results for unaided recall thus shows that scent does indeed enhance memory for verbal information (an additive effect) and that scent's (vs. picture's) effectiveness is greater in the longversus short-term.

\section{Incremental aided recall}

A similar repeated-measures MANOVA was conducted on incremental aided recall with scent, image, and time delay as factors. We obtain significant between-subject main effects for
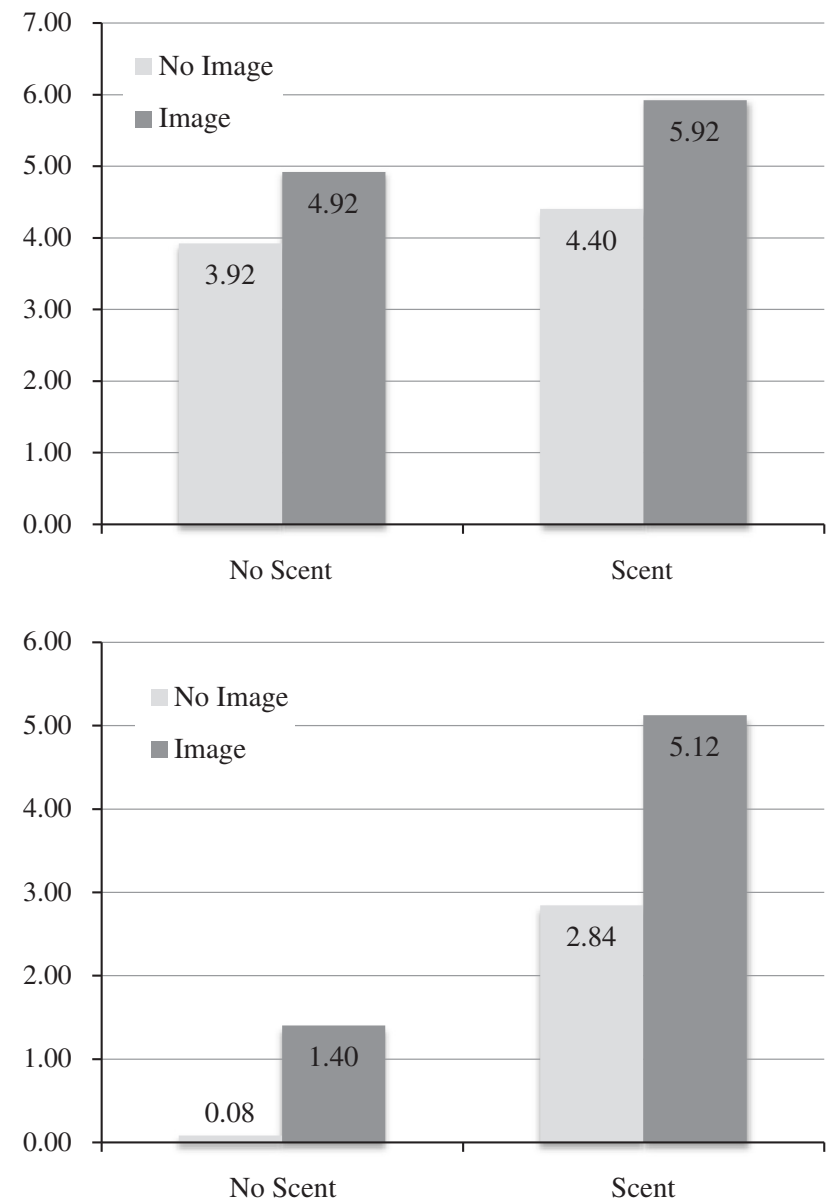

Fig. 2. Study 1: attributes recalled (unaided + incremental aided). Top: after short delay (5 minutes). Bottom: after long delay ( 2 weeks). scent $\left(F(1,96)=44.44, p<.0001, \eta^{2}=.32 ; M_{\mathrm{NoScent}}=0.20 \mathrm{vs}\right.$. $\left.M_{\text {Scent }}=1.03\right)$ and image $\left(F(1,96)=32.52, p<.0001, \eta^{2}=.25\right.$; $M_{\text {NoImage }}=0.26$ vs. $\left.\mathrm{M}_{\text {Image }}=0.97\right)$, as well as a significant interaction between the two $\left(F(1,96)=6.20, p<.05, \eta^{2}=.06\right)$.

We further explore the interaction between scent and image to see if there is a superadditive effect of scent and image on verbal memory. We conducted contrast tests comparing recall in the scented versus unscented conditions, with and without the floral image. Averaging across time delay, we find that image increased aided recall by less than one half of an item if scent was absent at the time of encoding $\left(F(1,96)=5.16, p<.05, M_{\text {NoScent } / \text { Nolmage }}=\right.$ $0.00 \mathrm{vs}$. $\left.M_{\text {NoScent/Image }}=0.40\right)$ versus by more than one full item if scent had been present at the time of encoding $(F(1,96)=33.59$, $p<.0001, M_{\text {Scent } / \text { NoImage }}=0.52$ vs. $\left.M_{\text {Scent/Image }}=1.54\right)$. In this context, which averages across time delays and involves reintroducing the sensory retrieval cues, we observe that scent effectively doubles the capacity of pictures to improve recall of verbal information. Thus, scent potentiates pictures' effect on recall and demonstrates a superadditive effect on verbal memory-providing preliminary support for H1. Next, we explore the impact of time delay on this pattern of results.

Within-subject effects revealed an interaction of scent and delay $\left(F(1,96)=6.20, p<.05, \eta^{2}=.06\right)$ and a marginally significant interaction of scent, image, and time delay $(F(1,96)=3.41, p<.10$, $\left.\eta^{2}=.03\right)$. A follow-up analysis shows that after a short time delay, scent $\left(F(1,96)=8.09, p<.005, \eta^{2}=.08 ; M_{\mathrm{NoScent}}=0.30 \mathrm{vs}\right.$. $\left.M_{\text {Scent }}=0.82\right)$ and image $\left(F(1,96)=13.84, p<.0001, \eta^{2}=.13\right.$; $M_{\text {NoImage }}=0.22$ vs. $\left.M_{\text {Image }}=0.90\right)$ both have significant effects on aided recall, but there is no interaction or synergistic effect evident. After a long time delay, we again find significant effects for scent $(F$ $(1,96)=45.44, p<.0001, \eta^{2}=.32 ; M_{\text {NoScent }}=0.10$ vs. $\left.M_{\text {Scent }}=1.24\right)$ and image $\left(F(1,96)=19.15, p<.0001, \eta^{2}=.17 ; M_{\text {NoImage }}=0.30 \mathrm{vs}\right.$. $\left.M_{\text {Image }}=1.04\right)$, as well as a significant scent $\times$ image interaction $(F$ $\left.(1,96)=10.19, p<.005, \eta^{2}=.10\right)$. After a long time delay, if there was no scent present at encoding, the floral image increased aided recall by just 0.2 items $\left(F(1,96)=0.70, p>.40, M_{\text {NoScent } / \text { NoImage }}=\right.$ 0.00 vs. $\left.M_{\text {NoScent/Image }}=0.20\right)$; however, if scent was present at encoding, image increased recall by over one full item $(F(1,96)=$ $28.68, p<.0001, M_{\text {Scent } / \text { Nolimage }}=0.60$ vs. $\left.M_{\text {Scent } / \text { Image }}=1.88\right)$. These results demonstrate that scent potentiates the effects of pictorial images on aided recall after a time delay (H1). Scent's potentiating effect of pictures on total recall can be seen in Fig. 2, where scent's ability to boost the effect of pictures on recall is much greater after a long (vs. short) time delay (seen in the relative height of the darker vs. lighter contiguous bars). 


\section{Additional analysis}

If it is true that scent has a superadditive effect on recall after a time delay because it activates the pictorial information stored in an imaginal code, we should find that scent increases memory for information presented pictorially in the ad, that is, recall related to the floral image (e.g., "fields of flowers," "yellow sunflowers," etc.). To test this idea, we analyzed more specifically recall related to the floral image. Thus, besides coding items for recall of the 13 verbal attributes and brand name, we also coded subjects' recall for the number of items that subjects remembered that related to the floral image (floral imagery means are reported in parentheses in Table 1).

We conducted a repeated-measures MANOVA on unaided and incremental aided recall of the number of items that subjects remembered that related to the floral image after a long time delay as a function of scent and image (Note: we conducted a similar analysis on recall after a short delay, and neither scent nor the scent $\times$ image interaction were significant). We obtain a significant effect for scent $\left(F(2,95)=21.83, p<.0001, \eta^{2}=.32\right)$, for image $\left(F(2,95)=10.75, p<.0001, \eta^{2}=.18\right)$, and for the interaction between the two $\left(F(2,95)=5.43, p<.0001, \eta^{2}=.10\right)$. Tests of between-subjects effects show that the floral image improved both the number of unaided $\left(F(1,96)=4.56, p<.05, \eta^{2}=.05\right.$, $M_{\text {NoImage }}=0.12$ vs. $\left.M_{\text {Image }}=0.30\right)$ and aided $(F(1,96)=16.82$, $p<.0001, \eta^{2}=.15, M_{\text {NoImage }}=0.20$ vs. $\left.M_{\text {Image }}=0.70\right)$ items recalled relating to the floral image, whereas scent improved only aided $\left(F(1,96)=40.92, p<.0001, \eta^{2}=.30, M_{\text {NoScent }}=0.06\right.$ vs. $\left.M_{\text {Scent }}=0.84\right)$ recall of such items. Importantly, the interaction between scent and image was significant for aided recall $(F$ $\left.(1,96)=9.71, p<.005, \eta^{2}=.09\right)$. Specifically, we find that incremental aided recall does not increase $\left(M_{\text {NoImage }}=.00\right.$ to $M_{\text {Image }}=.12, p>.45$ ) if a floral image was present at encoding but no scent; however, if scent was also present at encoding, the floral image increases aided recall of floral image items significantly $\left(M_{\text {NoImage }}=.40\right.$ to $\left.M_{\text {Image }}=1.28, p<.0001\right)$. Scent's ability to facilitate memory for pictorially presented information in the ad when presented with a sensorial retrieval cue after a long time delay thus supports the notion that the superadditive effects of scent on pictures are a reflection of scent's ability to activate information stored in imaginal codes.

\section{Discussion}

We find that pictorial input improves memory for verbal information, in accord with much prior research based on dual coding theory. We also find that olfactory input improves memory for verbal information, and we find this is true only after a time delay, a result reminiscent of odor recognition research. Scent can thus act like pictures to some extent, in terms of enhancing verbal memory, but the size of the scent versus picture effect differs over time, with scent more effective after a time delay.

Importantly, we find that scent potentiates the memoryenhancing effect of pictures when the sensory cues are reintroduced after a time delay. That is, superadditive effects emerge when scent appears to have its most potent effect on memory: after a time delay. The results show the interactive effects of scent and pictures effectively counteracting the normal effects of decay on memory performance. This pattern of results may be indicative of unique aspects of the multiple-stage memory consolidation processes associated with olfactory coding and the integration of these memory traces with information coded via the other senses. The synergistic effects of pictures and scent suggest that scent may not be stored in a completely independent memory code but may have the capacity to interact with the storage and later activation of information obtained via the other sensory modalities. The patterns of recall for items related to floral imagery support the notion that scent enhances pictures' facilitative effects on verbal recall by enhancing the visual imagery of the memory trace.

Interestingly, we found scent's potentiating effect on pictures occurred only when the scent was re-experienced, that is, when the consumer was able to re-experience some of the direct perceptual properties associated with the original promotional experience (MacInnis \& Price, 1987). When the scent was reintroduced to the consumer as a retrieval cue at a later date, it may have evoked sensory images of the original experience that helped the consumer to relive that experience across several sensory dimensions and reintegrate the various memory traces coded via the multiple senses. Paivio (2007, p. 66) has suggested that sensory cues with high imagery values activate concrete meanings of abstract associates and thus enhance recall. We found evidence that re-presenting the scent cue at retrieval significantly increased the ability to recall pictorially presented information in the ad (i.e., floral imagery).

The impact of scent on visual imagery is further explored in study 2, described next. Another goal of study 2 is to rule out a potential alternative explanation for the observed pattern of results. Is it possible that scent's enhancement of memory in study 1 was due merely to a difference in cognitive elaboration resulting from the inclusion of an additional sensory cue at stimulus exposure (i.e., at the time of information encoding; Meyers-Levy 1991)? Because we did not measure cognitive processing efforts in study 1 , this remains a potential alternative explanation. To rule out this possibility, we control for elaboration at encoding and manipulate only the type of sensory-based retrieval cue provided at the time of retrieval in study 2 . Thus, whereas in study 1 we manipulated the sensory stimulus cues in phase 1 (via the advertisement's stimulus properties), in study 2 we manipulate the stimulus cues only in phase 2 (via the provision of retrieval cues). If it is truly the amount of elaboration at the time of stimulus exposure that drove the results in study 1 (the alternative explanation), controlling for elaboration at exposure would produce only null results in study 2 .

\section{Study 2: sensory input manipulated at retrieval}

In this study, all participants received the same direct mailer print ad stimulus in phase 1 . The print ad consisted of a "fully loaded" stimulus in that it contained both the floral image as well as the rose/sandalwood scent. As in study 1, participants inspected the advertisement and evaluated the advertised product (to support the cover story). One week later, in phase 2, they were presented with a sensory retrieval cue and were asked to recall all they could about the advertised product. We varied sensory input only via the type of retrieval cue provided when memory is tested in this study. In phase 2, we also measured sensory imagery. 
Thus, the key differences between this study and study 1 are as follows: the sensory cues are manipulated at retrieval rather than at encoding; we measure recall only once (rather than twice) after a 1-week time delay (rather than after 5 minutes and 2 weeks); we focus only on aided recall (rather than both unaided and aided recall); and we also more directly measure sensory imagery - to see whether scent has the capacity to enhance both the olfactory and visual dimensions of imagery. Manipulating sensory input at retrieval (rather than at encoding) allows us to rule out the possibility of differential encoding at the time of stimulus exposure. Measuring only one form of memory at one point in time enables us to rule out the possibility of any carry-over effects from the within-subjects design used in study 1 .

\section{Sample and design}

One hundred forty undergraduates ( $80 \%$ female) were recruited and were given course credit for participation. The study had a 2 (retrieval cue contains floral image: yes, no) $\times 2$ (retrieval cue contains scent: yes, no) between-subjects design as a function of the type of retrieval cue provided at the time of memory testing. Each participant was randomly assigned to one of the four cells of the experimental design (retrieval cue with scent only, image only, both scent and image, or neither scent nor image).

\section{Stimulus materials}

The same full-color $8.5 \times 11$-inch direct mailer insert describing a hypothetical brand of moisturizer used in study 1 was used in this study in phase 1, with all participants seeing the same "fully loaded" print ad, which contained both the floral image and scent.

In phase 2 , when recall was tested exactly 1 week later, participants were provided with one of four types of retrieval cue. The retrieval cue consisted of a piece of paper with (or without) a reproduction of the floral visual imagery that appeared on the ad, and with (or without) the sandalwood/ rose scent contained in the ad.

\section{Procedure}

The procedure in phase 1 of study 2 was nearly identical to that of study 1. It involved asking participants to examine the moisturizer advertisement which was received in an envelope, and when done, to place the ad back in the envelope and answer several questions. They evaluated the advertised product on three 5-point items (bad/good, unappealing/appealing, low quality/high quality) - to support the cover story of the experiment - and were asked to return in 1 week for an unrelated study.

In phase 2, participants were instructed, "You examined an advertisement for a moisturizer product one week ago. We would like to ask you some questions about that product now." They were first told: "Please take out, look at, and smell the piece of paper in the ziplock bag attached to your envelope" and then answered several questions, which included providing the brand name of the product and everything else they could remember about the moisturizer. This provided the basis for our measurement of aided recall. Participants were then instructed to think about the advertised product and tell us "how easy it is to imagine" how the product: looks, smells, feels, sounds, tastes, each on a scale of $1=$ hard to imagine to $5=$ easy to imagine. Participants finally completed additional close-ended items to measure demographics, a hypothesis probe, and were thanked for participation. (Note: $93 \%$ of participants noticed the scent on the direct mailer in response to a direct question at the end of phase 2 ; however, none wrote in the open-ended hypothesis probe that they thought the purpose of the study was to test the effect of scent on memory, and thus, all participants were included in the analysis.).

\section{Results}

In this experiment, we measured aided recall as the sum of all correct items of information recalled when presented with the sensory retrieval cue (of 13 possible plus the brand name $=14$ total possible score; see Fig. 3). All coding was conducted by two independent coders and had a 93\% agreement rate. Any disagreements between the coders were resolved via discussion.

\section{Aided recall}

We conducted an ANOVA on aided recall as a function of the between subjects factors of retrieval cue scent (yes, no) and retrieval cue image (yes, no) plus a covariate for gender, since we included both males and females in this study. There were no effects of gender in any of the analyses and this variable is not discussed further. There was a significant main effect of scent $\left(F(1,135)=46.59, p<.0001, \eta^{2}=.26\right)$, demonstrating that a scented retrieval cue significantly increased the number of product attributes recalled $\left(M_{\text {NoScent }}=0.76\right.$ vs. $\left.M_{\text {Scent }}=2.19\right)$. Image was not significant $(p>.25)$. However, there was a significant interaction between scent and image $(F(1,135)=$ $\left.7.15, p<.01, \eta^{2}=.05\right)$. Inspection of the means showed that when the retrieval cue was unscented, the presence of the visual image in the retrieval cue did not significantly improve recall $\left(M_{\text {NoScent } / \text { NoImage }}=0.93\right.$ vs. $\left.M_{\text {NoScent/Image }}=0.60, p>.25\right)$; however, when the retrieval cue was scented, the presence of the visual image in the retrieval cue did improve recall $\left(M_{\text {Scent } / \text { NoImage }}=1.79\right.$ vs. $\left.M_{\text {Scent/Image }}=2.58, \mathrm{p}<.01\right)$. This result supports the superadditive effect of scent on pictures' ability to enhance memory, the effects of which are evident after a time delay (H1). The study's design also allows us to rule out the possibility that differences in elaboration at the time of encoding are driving the results.

\section{Imagery}

We next analyzed the five sensory dimensions of sensory imagery measured in phase 2 , namely, how easy it was to imagine how the product looks, smells, feels, sounds, and tastes. We first conducted a factor analysis with varimax rotation on these five items, and found that two factors accounted for $70.3 \%$ of the variation in response. The first factor contained looks (.81 loading) and smells (.82 loading); the second factor contained feels $(.74$ loading), sounds (.88 loading), and tastes (.86 loading). Thus, the ease with which participants could imagine how the product looked was highly correlated with the ease with which participants could imagine how the product smelled. This result provides some initial 
corroboration for our hypothesis that scents and pictures might exhibit interactive effects on sensory imagery and thus on the retrievability of information encoded via the imaginal code. We proceeded to analyze these two dimensions of sensory imagery further.

We conducted a MANOVA on the ease of imagining how the product looked and smelled at the time retrieval as a function of whether the retrieval cue contained scent (yes, no) and image (yes, no). The multivariate tests showed that only scent emerged significant $(F(2,135)=4.16, p<.05)$. The between-subjects effects tests showed that scent increased both the ease of imagining how the product smelled $(F(1,136)=$ $4.99, p<.05)$ as well as how it looked $(F(1,136)=6.33, p<.05)$. Specifically, it was easier to imagine how the product smelled $\left(M_{\text {Scent }}=4.13\right.$ vs. $\left.M_{\text {NoScent }}=3.72\right)$ and looked $\left(M_{\text {Scent }}=3.34\right.$ vs. $\left.M_{\text {NoScent }}=2.82\right)$ if the retrieval cue was scented. The evidence thus suggests that scent enhances not only olfactory but also visual imagery (H2). Here, we measured imagery with a different measure, from that used in study 1, providing additional support for the reason behind scent's ability to potentiate pictures' effects on recall-namely, its effect on visual imagery.

\section{Discussion}

In study 2, we again find that after a time delay, in this case, 1 week from ad exposure, scent enhances verbal recall and a scented retrieval cue potentiates the impact of pictures on retrieval. Unlike in study 1 , in study 2 we controlled for potential differences in information processing at the time of encoding by presenting all participants with an identical advertising stimulus in phase 1 . That is, here, all participants received the same "fully loaded" stimulus in the form of a scented ad with floral images at the time of encoding. The only manipulation consisted of the type of retrieval cue provided at the time of attempted recall: one that contained either scent, a floral image, both, or neither. This design allowed us to rule out the possibility that the positive effects of scent on recall are due merely to increased elaboration at the time of stimulus exposure.

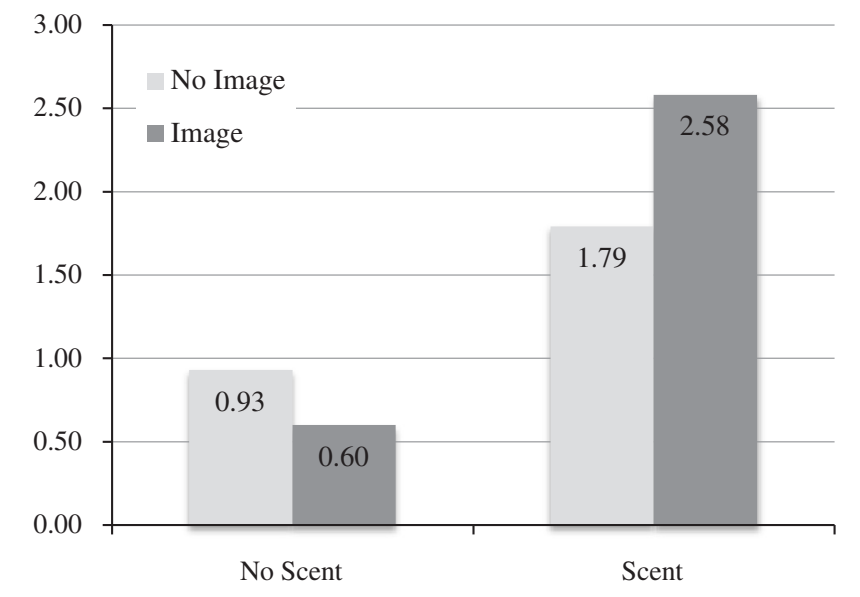

Fig. 3. Study 2: product attributes recalled (aided). After long delay (1 week).
We also obtained additional process evidence in the form of the multisensorial dimensions of sensory imagery. These results show that scent may help to concretize the memory trace by increasing the ease with which it can not only be smelled but also seen in one's mind-scent increased not only how easy it was to imagine how the product smelled but also how easy it was to imagine how the product looked. The capacity of scent to impact imagery related to the other sensory modalities may explain its superadditive effects on pictorial input. These results provide additional support for the multisensorial imagery value of scent as a retrieval cue, wherein the scent-based cue helps to reinstitute the entire contents of the memory episode, thus serving as what Paivio referred to as a "conceptual peg" (Paivio, 2007). Thus, scent may almost act like a picture, in terms of its ability to aid a person's ability to remember what the product looked like.

\section{General discussion}

Visual images have been likened to a "picture in the head" (Wyer et al., 2008a,b) and much prior research has shown that pictures enhance verbal memory. Here, we find that scents may act as a "smell in the head" that similarly enhance recall of verbal information and also have the capacity to potentiate the effect of pictures on recall. We found that visual images, which Barsalou (1999) referred to as the "cognitive glue" for linking sensory inputs, may be made even stickier with scent. We refer to this as the potentiating effect of scent on pictures' ability to enhance memory for verbal information.

Our findings extend dual coding theory beyond the domain of verbal and pictorial input to encompass olfactory input as well. We found that scents enhance the retrievability of verbal information, similar to pictures; however, we found that scent also exhibits some unique characteristics. Specifically, we found that, unlike pictures, scent has greater effects on memory after a time delay. Our results therefore support the idea that there may be multiple stages involved in the memory consolidation process for olfactory information.

Our results do not seem to support the notion that scents are stored in an entirely independent olfactory code (Lyman \& McDaniel, 1990). Instead, we found that smelling a scent previously encountered increases the ease with which one cannot only "smell" but "see" the object to be recalled. Thus, scent may interact with other sensory aspects of the imagery value of the memory trace (e.g., visual, tactile, etc.) and activate or potentiate the memory enhancing effects of these sensory inputs. With retrieval cues, scents and pictures were found to act synergistically at improving recall, exhibiting superadditive effects - going beyond the well-known additivity hypothesis of DCT.

The results reported here should be of interest to a growing number of marketers, as the use of olfaction, or the sense of smell, has emerged in a variety of novel marketplace contexts: scent released near outdoor advertisements in bus shelters (Gordon, 2006), scents stored in microencapsulated form in direct mailers (Pfanner, 2007), and scent emitted in museums (Aggleton \& Waskett, 1999), movies theatres (Elliott, 2007), and banks (McGregor, 2008). Our results demonstrate very 
clearly that scent tends to enhance recall and can even help other sensory inputs to enhance recall as well.

We found the potentiating effects of scent on pictures occurred after a time delay, which is what managers are arguably most concerned with, since they want the ad information to be remembered when the consumer decides to shop, which is unlikely to be immediately after seeing the ad. With a scent retrieval cue (e.g., a Laura Ashley, Benetton, or Marriott signature scent that is emitted in a cinema ad as well as in their store/hotel), consumers are able to utilize the pictorial information in the ads even more to enhance memory after a long period from ad exposure.

Future studies could explore the impact of haptic (i.e., touch; Peck \& Childers, 2003) and taste-based input on memory for verbal information, with and without the presence of pictorial input. Would similar superadditive effects be observed, or are there specific characteristics associated with olfactory processing that make it unique in this regard? It would also be interesting in future research to explore whether individual differences moderate the effects observed here. For example, would those who exhibit a chronic disposition to process information visually rather than verbally demonstrate enhanced synergistic effects of multimodality processing (Childers, Houston \& Heckler, 1985)? The set of questions to be explored regarding the interactive effects of multisensorial inputs is large and fascinating.

\section{References}

Aggleton, J. P., \& Waskett, L. (1999). The ability of odours to serve as statedependent cues for real-world memories: Can Viking smells aid the recall of Viking experiences? British Journal of Psychology, 90(1), 1-7.

Anderson, J. R., \& Bower, G. H. (1973). Human associative memory. Washington, DC: Winston.

Bagozzi, R. P. (2008). Some insights on visual and verbal processing strategies. Journal of Consumer Psychology, 18(4), 258-263.

Barsalou, L. W. (1999). Flexibility, structure, and linguistic vagary in concepts: Manifestations of a compositional system of perceptual symbols. In A. F. Collins, S. E. Gathercole, M. A. Conway, \& P. E. Morris (Eds.), Theories of memory (pp. 29-102). Hillsdale, NJ: Erlbaum.

Begg, I. (1972). Recall of meaningful phrases. Journal of Verbal Learning and Verbal Behavior, 11, 431-439.

Bensafi, M., Porter, J., Pouliot, S., Mainland, J., Johnson, B., Zelano, C., et al. (2003). Olfactomotor activity during imagery mimics that during perception. Nature Neuroscience, 6(11), 1142-1144.

Childers, T. L., \& Jiang, Y. (2008). Neurobiological perspectives on the nature of visual and verbal processes. Journal of Consumer Psychology, 18(4), 264-269.

Childers, T. L., Houston, M. J., \& Heckler, S. E. (1985). Measurement of individual differences in visual versus verbal information processing. Journal of Consumer Research, 12, 125-134.

Ebbinghaus, H. (1885). Uber das Gedachtnis [On memory]. Leipzig: Dunker.

Elliott, S. (2007, September). Movies soon really will smell; The one, in an ad, like a cake. New York Times, 3, C6.

Engen, T. (1987). Remembering odors and their names. American Scientist, 75, 497-503.

Engen, T., Ross, B. E., \& Eimas, P. D. (1973). Short-term memory of odors. Journal of Experimental Psychology, 99(2), 222-225.

Engen, T., \& Ross, B. M. (1973). Long-term memory of odors with and without verbal descriptions. Journal of Experimental Psychology, 100(2), 221-227.
Gerber, B., \& Menzel, R. (2000). Contextual modulation of memory consolidation. Learning and Memory, 7(3), 151-158.

Gordon, R. (2006, December). At bus stops, scents of dissent. The Philadelphia Inquirer, 17, A29.

Heckler, S. E., \& Childers, T. L. (1992). The role of expectancy and relevancy in memory for verbal and visual information: What is incongruency? Journal of Consumer Research, 18, 475-492.

Houston, M. J., Childers, T. L., \& Heckler, S. E. (1987). The effects of pictureword consistency on the elaborative processing of print advertisements. Journal of Marketing Research, 24, 359-369.

Lyman, B. J., \& McDaniel, M. A. (1990). Memory of odors and odor names: Modalities of elaboration and imagery. Journal of Experimental Psychology: Learning, Memory and Cognition, 16(4), 656-664.

MacInnis, D. J., \& Price, L. L. (1987). The role of imagery in information processing: Review and extensions. Journal of Consumer Research, 13, 473-491.

McGregor, J. (2008, April). The sweet smell of...deposits. BusinessWeek, 7, 26.

McKeever, W. F., \& Hulling, M. D. (1971). Lateral dominance in tachistoscopic word recognition performances obtained with simultaneous bilateral input. Neuropsychologia, 9(1), 15-20.

Meyers-Levy, J. (1991). Elaborating on elaboration: The distinction between relational and item specific elaboration. Journal of Consumer Research, 18, 358-367.

Miniard, P., Bhatla, W., Lord, K. L., Dickson, P. R., \& Unnava, H. R. (1991). Picture-based persuasion processes and the moderating role of involvement. Journal of Consumer Research, 18(1), 92-107.

Paivio, A. (1969). Mental imagery in associative learning and memory. Psychological Review, 76(3), 241-263.

Paivio, A. (1971). Imagery and verbal processes. New York: Holt, Rinehart, and Winston.

Paivio, A. (1986). Mental representations: A dual coding approach. Oxford, England: Oxford University Press.

Paivio, A. (2007). Mind and its evolution: A dual coding theoretical interpretation. Mahwah, NJ: Lawrence Erlbaum Associates. Inc..

Peck, J., \& Childers, T. L. (2003). Individual differences in haptic information processing: On the development, validation, and use of the 'Need for Touch' scale. Journal of Consumer Research, 30(3), 430-442.

Pfanner, E. (2007, November 15). Sending a scent by snail mail (pp. C9). : The New York Times.

Rizzolatti, G., Umilta, C., \& Berlucchi, G. (1971). Opposite superiorities of the right and left cerebral hemispheres in discriminative reaction time to physiognomical and alphabetical material. Brain, 94, 431-442.

Sanchez-Andrade, G., James, B. M., \& Kendrick, K. M. (2005, Oct.). Neural encoding of olfactory recognition memory. Journal of Reproductive Development, 51(5), 547-558.

Shepard, R. N. (1967). Recognition memory for words, sentences and pictures. Journal of Verbal Learning and Verbal Behavior, 6, 156-163.

Spangenberg, E. R., Crowley, A. E., \& Henderson, P. W. (1996). Improving the store environment: Do olfactory cues affect evaluations and behaviors? Journal of Marketing, 60(4), 67-80.

Spangenberg, E. R., Sprott, D. E., Grohmann, B., \& Tracy, D. L. (2006). Gender-congruent ambient scent influences on approach and avoidance behaviors in a retail store. Journal of Business Research, 59, 1281-1287.

Thompson, V., \& Paivio, A. (1994). Memory for pictures and sounds: Independence of auditory and visual codes. Canadian Journal of Experimental Psychology, 48, 380-398.

Wyer, R. S., Hung, I. W., \& Jiang, Y. (2008). Visual and verbal information processing in a consumer context: Further considerations. Journal of Consumer Psychology, 18(4), 276-280.

Wyer, R. S., Hung, I. W., \& Jiang, Y. (2008). Visual and verbal processing strategies in comprehension and judgment. Journal of Consumer Psychology, $18(4), 244-257$.

Zucco, G. M. (2003). Odor memory: The unique nature of memory system. In Martina Plumacher, \& Peter Holz (Eds.), Speaking of colors and odors (pp. 155-166). Philadelphia, PA: John Benajamins Publishing Company. 